\title{
22q13 Microduplication Syndrome in Siblings with Mild Clinical Phenotype: Broadening the Clinical and Behavioral Spectrum
}

\author{
Anikó Ujfalusia Orsolya Nagy ${ }^{a}$ b Beáta Bessenyei ${ }^{a} \quad$ Györgyi Lente ${ }^{c}$ \\ Irén Kántor ${ }^{d}$ Ádám J. Borbélye Katalin Szakszon ${ }^{f}$ \\ ${ }^{a}$ Division of Clinical Genetics, Department of Laboratory Medicine, Faculty of Medicine, University of Debrecen, \\ Debrecen, Hungary; ${ }^{b}$ Doctoral School of Clinical Medicine, University of Debrecen, Debrecen, Hungary; \\ ${ }^{c}$ Department of Psychology, Rehabilitation Centre for Children, Debrecen, Hungary; ${ }^{d}$ Department of Pediatrics, \\ Jósa András County and Teaching Hospital, Nyíregyháza, Hungary; ${ }^{e}$ Department of Psychiatry, Sántha Kálmán \\ Special Hospital, Nagykálló, Hungary; ' Institute of Pediatrics, Faculty of Medicine, University of Debrecen, Debrecen, \\ Hungary
}

\section{Established Facts}

- Terminal deletion of chromosome $22 \mathrm{q}$ is a rare disorder with variable degree of developmental delay and association of morphological symptoms. It may arise as a de novo event or, less frequently, from parental balanced reciprocal translocation or a pericentric inversion of chromosome 22 .

- Involvement of SHANK3 in the duplicated segment is crucial in the emerging neurobehavioral phenotype.

\section{Novel Insights}

- Familial reoccurence presented in this paper is extremely rarely reported.

- Our patients have unusually mild clinical symptoms - they share very similar morphological features, neither patient has microcephaly, they both have borderline intellect, and they have the exact same chromosomal breakpoints on array CGH.

- SHANK3 is involved in the microduplication, and the mother is a carrier of a pericentric inversion of chromosome 22.

\section{Keywords}

Array CGH · Distal trisomy $22 \mathrm{q} \cdot$ Pericentric inversion .

22 qter $\cdot 22 q 13$

\section{Abstract}

Distal duplication $22 q(22 q 13.3 q$ ter $)$ is a rare condition with only 24 cases described so far. Parental balanced reciprocal translocations and pericentric inversions involving chromo- some 22 predispose to the conception of an unbalanced offspring and are more frequently reported than de novo events. The clinical phenotype of patients is highly variable and does not necessarily correlate with the extent of the duplicated segment. Short stature, microcephaly, hypertelorism, cleft lip or palate, low-set ears, and intellectual disability seem to be the most consistent features. Familial reoccurrence is extremely rarely reported. Here, we report 2 siblings with a 22q13.3qter duplication detected by array

\section{KARGER}

(C) 2020 S. Karger AG, Basel

karger@karger.com www.karger.com/msy
Katalin Szakszon

Institute of Pediatrics, Faculty of Medicine University of Debrecen

Nagyerdei krt. 98, HU-4032 Debrecen (Hungary)

szakszon.katalin@med.unideb.hu 
$\mathrm{CGH}$; their mother is a carrier of a pericentric inversion in chromosome 22. Their relatively mild phenotype and identical chromosomal breakpoints as well as duplication size are unique. This is the first case described so far.

(c) 2020 S. Karger AG, Basel

Duplication of the distal long arm of chromosome 22 (22q13qter) is a rare cytogenetic anomaly, resulting in variable degrees of developmental delay and dysmorphism with or without obvious neuropsychiatric symptoms in the affected individuals. To date, only 24 cases have been published, the majority of which were identified by conventional cytogenetics and FISH [Fryns et al., 1980; Schinzel, 1981; Bendel et al., 1982; Biesecker et al., 1995; Wieczorek et al., 1998; Petek et al., 2000; Boyd et al., 2005; Hou, 2005; Feenstra et al., 2006; Failla et al., 2007; Okamoto et al., 2007; Peeters et al., 2008; Wu et al., 2010; Jafri et al., 2011; Han et al., 2013; Ahn et al., 2014; Magri et al., 2015; Chen et al., 2017; Johannessen et al., 2019]. Two of the above patients were identified in the context of large studies designed for unexplained developmental delay and schizophrenia with limited phenotype description [Wu et al., 2010; Ahn et al., 2014]. Han et al. [2013] reported the 2 smallest duplications of 22q13 so far.

The molecular cytogenetic classification of pure distal 22q duplications was proposed by Feenstra et al. [2006] distinguishing 4 groups: large duplication (22q12qter), intermediate (22q13.1qter), small (22q13.2qter), and smallest duplication (22q13.3qter). They also provided an overview of patients from the literature and their own cases. They concluded that patients having a duplication extending from $22 \mathrm{q} 13$ to qter show most clinical problems and have a lower survival rate, but even patients with assumingly comparable duplications show great variations, and comparison between them is difficult. The most consistent features are pre-and postnatal growth retardation, cleft palate with or without cleft lip, micrognathia, microcephaly, hypertelorism, low-set ears, congenital heart defect, renal and genital anomalies as well as hypotonia. In some cases, decreased life expectancy is reported [Feenstra et al., 2006].

The majority of cases arise from parental balanced translocations, a smaller proportion of patients are de novo, or the offspring of a parent carries a pericentric inversion or insertion in chromosome 22. Jafri et al. [2011] demonstrated that during homologous recombination, commonly occurring in the process of meiosis, a parent with an inverted chromosome 22 has likelihood to produce a gamete with a rearranged form of the inverted chromosome 22. This rearranged form already contains either a deletion or duplication, resulting in the conception of an unbalanced offspring after fertilization [Jafri et al., 2011].

Magri et al. [2015] assessed the clinical and cytogenetic/molecular cytogenetic properties of 10 patients with duplications distal from 22q13 - the intermediate, small, and smallest terminal duplications - and explained the possible molecular mechanism involving a break-induced replication as part of a nonreciprocal translocation event, leading to a de novo unbalanced translocation of the distal part of chromosome 22. They also pointed out that patients with apparently the same duplicated region show a wide spectrum of phenotypic variations; part of these differences may be due to the different genetic background in which the duplications arise, but also to the different resolution of methods used to evaluate the extent of duplications [Magri et al., 2015]. The 6 patients with a molecular characterization of the breakpoints and array CGH analysis are those reported by Failla et al. [2007], Okamoto et al. [2007], Peeters et al. [2008], Magri et al. [2015], Chen et al. [2017], and Johannessen et al. [2019].

Here, we present 2 siblings with a rare 22q13qter duplication and only mild symptoms, detected by array CGH and FISH, whose mother is a carrier of a pericentric inversion of chromosome 22.

\section{Case Presentations and Results}

\section{Patient 1}

Patient 1 was born at term as the 3rd child of healthy, nonconsanguineous Caucasian parents, with normal weight $(2,750 \mathrm{~g} ; 10$ th percentile) and length $(49 \mathrm{~cm}$; 25 th percentile); occipitofrontal circumference (OFC) was not recorded. The mother and father are both of normal intellect and work for a living. The first child of the parents is a 24 -year-old, clinically healthy male, who works as a basic informatics educator, teaching computer skills to the elderly. The second child is patient 2 , an affected male. The mother had a brother, who - to her knowledge - was delivered by forceps, was severely mentally disabled, and lived in a special institution until he died in a fire accident at 26 years of age in the 1990s. He was nonverbal, tall statured, and had a long chin. No biological material was available.

Patient 1, an 18-year-old young woman upon the first examination, was referred to genetic counseling because of obesity, mild developmental delay, secondary amenorrhea, and minor morphological anomalies. Age at menarche was 16 years with only 1 menstruation in the following 2 years. At 16, her height was $158 \mathrm{~cm}$ (10th percentile), weight $109 \mathrm{~kg}$ ( $27 \mathrm{~kg}$, $>97$ th percentile), her BMI was 43.7. At 19 years, her OFC was $55 \mathrm{~cm}$ (50th percentile). There were striae on the skin of the lower abdomen, and acanthosis nigricans on the neck. Prader-Willi syndrome was specifically asked 

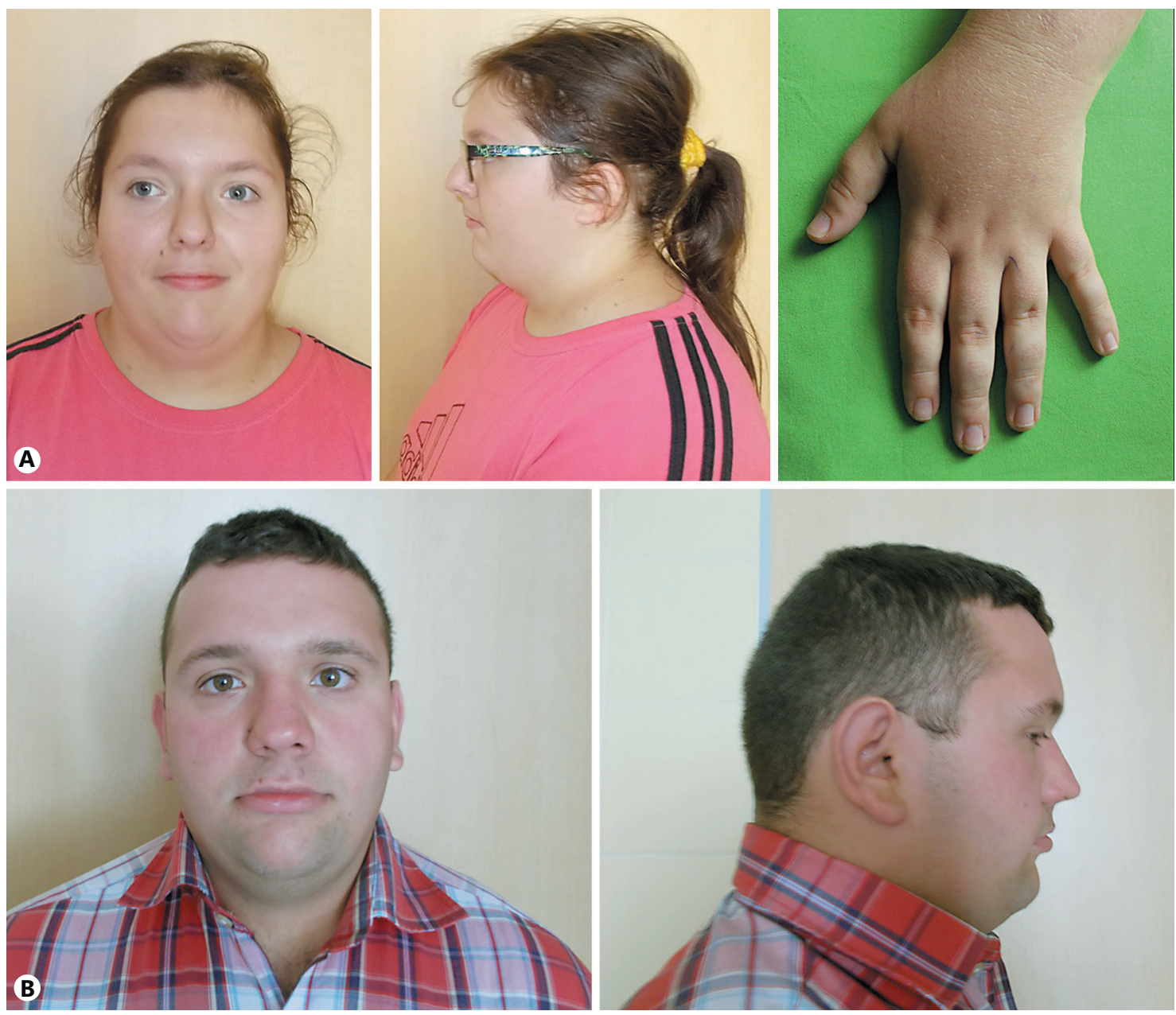

Fig. 1. A Patient 1 showing hypertelorism, divergent strabismus, broad eyebrows, high nasal bridge, bulbous tip of the nose, posteriorly low-inserted columella, small hands with tapering fingers, and an overall friendly nature. B Patient 2, brother of patient 1, with similar facial features. In addition, he has a deviated septum and more pronounced downslanting palpebral fissures.

to be ruled out by the endocrinologist, although the mental status and face did not resemble Prader-Willi syndrome. The patient had insulin resistance with normal fasting glucose, so dietary restrictions were prescribed. Central and peripheral sexual hormones were normal, subclinical hypothyreoidism was detected, sTSH: $8.24 \mathrm{mU} / \mathrm{L}$ (Ref: $0.3-4.2 \mathrm{mU} / \mathrm{L}$ ), and corrected with L-thyroxin. Gynecological examination revealed a normal uterus and ovaries. Brain MRI was normal. Bone age was equal to chronological age.

A detailed psychological examination revealed early developmental delay - the patient achieved independent walking at 22 months - and a borderline intellect using the Wechsler Adult Intelligence Scale with uneven performance levels: verbal comprehension index was 93 (average), perceptual organization 69, processing speed 68 (both below normal), working memory 74, with a full-scale IQ of 72. She showed signs of performance anxiety, but her task awareness was above average. During the test, she replaced the more difficult, not yet automatized mathematical operations such as multiplication and division by simply adding and subtract- ing but came to correct results. No behavioral problems were obvious; she had a conventional, open, and friendly personality. According to the psychological evaluation, the patient compensated her learning difficulties with good verbal skills, other successful compensational strategies, and sedulity. She was able to earn a high-school degree with satisfactory grades.

Her menstruation cycle returned to normal by the age of 18 years without further medical interference.

Morphologically, the patient had hypertelorism, mildly downslanting palpebral fissures, divergent strabismus, and broad eyebrows. The nasal bridge was high, and the nose was prominent, with posteriorly low-inserted columella and a bulbous tip. She had a long philtrum and thin upper lip with downturned corners of the mouth (Fig. 1A). Her hands and feet were small, the fingers distally tapering with normal bone structure on X-ray (Fig. 1B). Table 1 shows an overview of the clinical features of the 2 patients, based on the phenotypic assessment previously used by Feenstra et al. [2006] and Magri et al. [2015]. 
Table 1. Overview of clinical features of our patients with distal 22q duplications

\begin{tabular}{|c|c|c|}
\hline & Patient 1 & Patient 2 \\
\hline Duplicated region & 22q13.3qter & 22q13.3qter \\
\hline Gender & Female & Male \\
\hline Age at first referral & 18 years & 22 years \\
\hline \multicolumn{3}{|l|}{ Neurological features } \\
\hline Developmental delay & Yes & Yes, noted after 3 years of age \\
\hline Seizures & No & Yes, fever-associated \\
\hline Intelligence & Borderline (overall IQ 76) & Borderline (overall IQ 79) \\
\hline Microcephaly & No & No \\
\hline Brain structural abnormality & No & No \\
\hline \multicolumn{3}{|l|}{ Facial features } \\
\hline Sparse, fine hair & Yes & No \\
\hline Hypertelorism & Yes & Yes \\
\hline Palpebral fissures & Downslanted & Downslanted \\
\hline Strabism & Yes (divergent) & Yes (divergent) \\
\hline Wide/high nasal bridge & Yes & Yes \\
\hline Low-set ears & Yes & No \\
\hline Dysplastic ears & No & No \\
\hline Cleft lip/palate & No & No \\
\hline Long philtrum & Yes & Yes \\
\hline Retrognathia & No & No \\
\hline \multicolumn{3}{|l|}{ Skeletal features } \\
\hline Short neck & Yes & Yes \\
\hline Short stature & No & No \\
\hline Obesity & Yes & Yes \\
\hline Visceral anomaly (renal, cardiac, genital) & No & No \\
\hline Psychiatric problems & No & Yes \\
\hline Limbs & Small hands & Normal \\
\hline
\end{tabular}

G-banding revealed a normal female karyotype. The facial features and the mild developmental delay raised the suspicion of DiGeorge syndrome. Therefore, 22q11.2/22q13.3 FISH was performed (DiGeorge/VCFS TUPLE1/22q13 probe mix, Cytocell, Rainbow Scientific Inc., Windsor, CT, USA). However, instead of monosomy 22q11.2, trisomy of the 22q13.3 region was detected. Analysis of metaphase chromosomes revealed that one of the chromosomes 22 contains two 22q13.3 regions, one on the short arm and one on the terminal end of the long arm (Fig. 2A). Using array CGH (Affymetrix CytoScan $750 \mathrm{~K}$ and Affymetrix Chromosome Analysis Suite (ChAS) v2.0 Software, Affymetrix, Thermo Fisher Scientific, Waltham, MA, USA), a duplication of chromosome 22q13.31qter was identified, spanning 3,327 $\mathrm{kb}$ and 45 genes of which 30 are OMIM genes. SHANK3 was encompassed in the trisomy (Fig. 2C). The exact gene content of the duplication, identical in the siblings, with OMIM numbers is as follows: FAM19A5 (617499), BRD1 (604589), ZBED4 (612552), ALG12 (607144), CRELD2 (607171), PIM3 (610580), IL17REL (613414), MLC1 (605908), MOV10L1 (605794), PANX2 (608421), SELENOO (607917), TUBGCP6 (610053), HDAC10 (608544), MAPK12 (602399), MAPK11 (602898), PLXNB2 (604293), PPP6R2 (610877), SBF1 (603560), ADM2 (608682), MIOX (606774), NCAPH2 (611230), SCO2 (604272), TYMP (131222), SYCE3 (615775), CPT1B (601987), CHKB (612395), MAPK8IP2 (607755), ARSA (607574), SHANK3 (606230), and ACR (102480). The patient's molecular karyotype was: $\operatorname{arr}[$ hg19] 22q13.3 1q13.33(47,870,362-51,197,766)×3.

\section{Patient 2}

The brother is the 2nd term-born child of the parents with a weight of $3,100 \mathrm{~g}(10 \mathrm{th}-25 \mathrm{th}$ percentile) and length of $50 \mathrm{~cm}$ (25th percentile). OFC was not recorded, but there was no suspicion of microcephaly. According to the mother's memories, he seemed to be developing normally until 3 years of age, when febrile seizures developed, reoccurring several times until the age of 5 years. On 2 occasions, the convulsions were life-threatening and required cardiopulmonary resuscitation. He received lamotrigine, later carbamazepine therapy. He was noted to be a reserved child in kindergarten, not engaging in activities with peers. Challenging behavior developed later in his school years: he often lost interest in his classes and walked out without permission, dropping into other classrooms to chat with schoolmates. He went to normal primary school but needed extra support and education to achieve his degrees. Basic grammatical, mathematical and literature skills have been acquired. Dyslexia and dysgraphia manifested in learning difficulties, but he graduated from an evening high-school with satisfactory grades. He now works as a temporary employee in a restaurant.

He was diagnosed with bipolar affective disorder, and agomelatine and aripiprazole were prescribed - the latter was omitted 

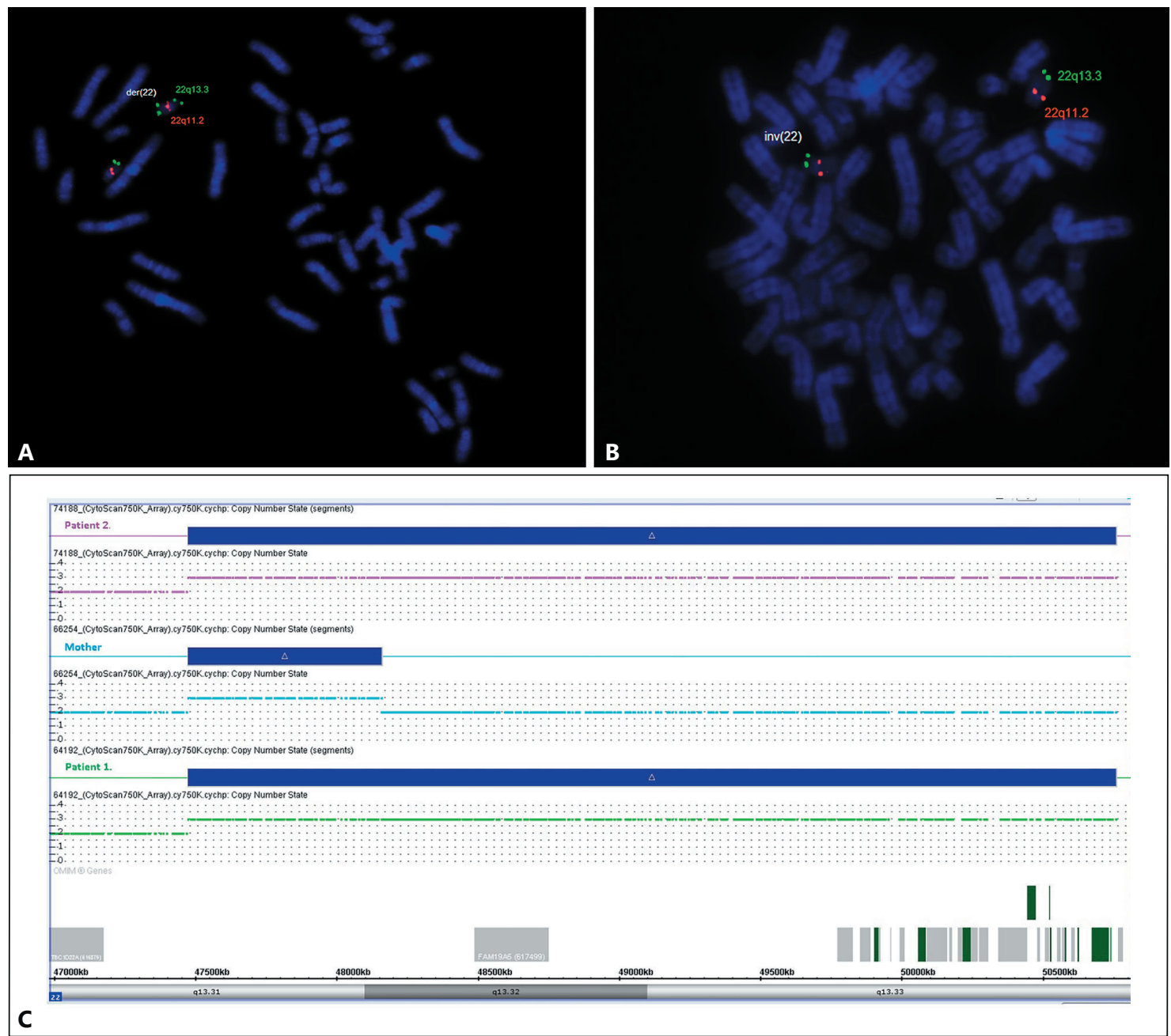

Fig. 2. A FISH on metaphase spread of patient 1 using locus specific probe for 22q11.2 (TUPLE1; red) and 22q13.3 (N85A3; green). The latter covers the telomeric end of the SHANK3 gene, allowing the identification of the most distal 22q13.3 rearrangements. The abnormal chromosome 22 contains two $22 \mathrm{q} 13.3$ signals. B FISH on metaphase spread of the mother using locus specific probe for 22q11.2 (TUPLE1; red) and 22q13.3 (N85A3; green). The inv(22) shows the inverted chromosome 22 and relo- cation of the 22q13.3 region to the short arm. C Array CGH of the probands and the mother using Affymetrix $750 \mathrm{~K}$ platform. The analysis shows the identical duplication (blue bars) of 3,327 kb involving the 22q13.31q13.33 region in patient 1 and 2 , with the breakpoints falling between 47,870,362 and 51,197,766. The smaller duplication detected in the mother's sample (middle) contains no OMIM genes. Dashed lines represent copy number statuses. because of fatigue, which he attributed to his medication. He has mild sleep disturbance, low frustration tolerance and occasional temper tantrums. Apart from this, he still likes to play with toys that are inappropriate to his age and comprehension, such as toy cars and tying knots on strings. His overall IQ at 18 years of age was 79; verbal IQ was 81, and performance 79 . His head circumference was $59 \mathrm{~cm}$ (90th percentile).

Cytogenetic analysis of peripheral blood showed a normal male karyotype. FISH study with a DiGeorge-specific probe mix confirmed the trisomy of the $22 \mathrm{q} 13.3$ region with the same signal pattern as in his sister. Array CGH was also performed; the breakpoints and the size of the duplicated 22q13.3 region were identical to his sister's duplication (Fig. 2C). Parental FISH testing clearly revealed a pericentric inversion of chromosome 22 with breakpoints $22 \mathrm{p} 13$ and $22 \mathrm{q} 13$ in the mother with the following karyotype: 46,XX.ish inv(22)(p13q13.3)(p13)(N85A3+)(q13.3) (N85A3-) (Fig. 2B). Based on the mother's FISH result, the final karyotypes of the probands were: 46,XX.ish $\operatorname{rec}(22) \operatorname{dup}(22 \mathrm{q} 13.3)$ inv(22)(p13q13)mat and 46,XY.ish rec(22)dup(22q13.3)inv(22) (p13q13)mat. Array CGH performed on the mother's DNA sample showed a small duplication $(685 \mathrm{~kb}$, breakpoints $47,474,613$ and $48,159,741)$ at the proximal breakpoint of the duplicated region detected in the probands (Fig. 2C). Presumably, this segment was duplicated as part of the process that generated the inversion. 
Based on the gene content (no OMIM genes) and the literature data, this duplication does not have phenotypic consequences.

The healthy male sibling's FISH testing revealed a normal signal pattern; he was not a carrier of a pericentric inversion either.

\section{Discussion}

Pure duplications of chromosome 22q, without concomitant aneusomies of other chromosomes, are exceedingly rare. Underreporting of mild cases may create a selective bias, so the true incidence of the condition remains unknown. Array CGH is a powerful tool in identifying causal copy number changes in patients with intellectual disability; it is considered to be useful even in cases of mild mental retardation [Liang et al., 2008; Coutton et al., 2015].

Most of the reported distal 22q trisomy cases were extensively studied, and the clinical features were overviewed in details by Feenstra et al. [2006] and Magri et al. [2015], latter focusing on duplications distal from 22q13. The cases described by Pramparo et al. [2008], Shimojima et al. [2009], Samanich et al. [2012], and Rahikkala et al. [2013] should be handled separately - their patients having small interstitial duplications more proximally (22q13.1 to q13.31), with overall more severe phenotypes. The frequency of pericentric inversions, excluding the common/polymorphic inversions, is estimated to be $0.12-0.7 \%$ [Gardner et al., 2011]. According to Koolen et al. [2005] the presence of recurrent proximal breakpoints at $22 \mathrm{q} 13$ indicates that these specific regions are prone to recombination that may lead to translocations, inversions, duplications, and deletions. Pericentric inversions are balanced rearrangements without phenotypic consequence; however, through recombination between the normal and the inverted allele, a parent with an inversion may transmit an unbalanced, rearranged form of the inverted chromosome to the offspring.

In this study, we reported the familial recurrence of terminal 22q duplication with 2 affected siblings originating from maternal pericentric inversion in chromosome 22 (p13q13.3). The breakpoints and the size of the detected duplication were identical in patient 1 and patient 2 , suggesting the same sequence and course of recombination events between the inverted and the normal maternal homologue of chromosome 22. The small duplication found at the breakpoint of 22q13.3 in the maternal chromosome 22 , partially overlapping with the proximal breakpoints of the 22q duplication in her children, is likely the result of recombination within the inversion loop.
Peeters et al. [2008] found evidence for a consistent clinical presentation in 22qter duplication: mild to moderate mental retardation, microcephaly, and similar mild dysmorphic features. Indeed, an overview of the cases in the literature, except for those published by Schinzel [1981], Jafri et al. [2011], and Han et al. [2013], report microcephaly. In our report, both patient 1 and 2 have mild dysmorphic features, such as hypertelorism, divergent strabismus, high nasal bridge, and a bulbous nose tip; they both developed obesity and learning difficulties. Yet, unlike most patients with distal terminal duplications, they both have normal height and head circumference, and borderline intellect, albeit with the help of longterm therapies. The phenotype seems milder in the young woman than in her brother, as she has no behavioral or adaptive problems. In their recent paper, Han et al. [2013] pointed out features that are the consequence of SHANK3 overexpression: hyperphagia, seizures, reduced social interactions, and manic-like behavior. They reported 2 patients with the smallest duplications so far, yet the neurobehavioral and psychiatric symptoms were quite prominent. In our case, obesity in both patients and the neuropsychiatric symptoms of patient 2 - maladaptive behavior, epilepsy, repetitive monotonous playing, and bipolar affective disorder - are highly reminiscent of features reported by Han et al. [2013]. Epilepsy as a clinical consequence of SHANK3 overexpression was also reported by Jin et al. [2018]. We assume that a gene dosage effect originating from the duplication of SHANK3 in our patients is responsible for the overlapping phenotype, yet recent literature points out the modifying effect of posttranscriptional regulation of the SHANK3 expression [Choi et al., 2015].

In conclusion, our report supports the observation of previous authors that clinical features in the 22qter duplication syndromes show great variability, and the size of the duplicated segment does not always correlate with the phenotypic severity. Our patients are 2 of the very few published cases of familial recurrence of 22qter duplication syndrome characterized by array CGH, and they represent unusually mild phenotypes without microcephaly, yet with detectable neuropsychiatric symptoms in one of them.

\section{Statement of Ethics}

Written informed consent for genetic testing and publication of photo material was obtained from all individuals. Although the tests were performed on a diagnostic purpose, ethical approval was also obtained (28676-7/2017/EÜIG). 


\section{Disclosure Statement}

The authors have no conflicts of interest to declare.

\section{Funding Sources}

This study was supported by the Ministry of National Economy, Hungary (GINOP-2.3.2-15-2016-00039).

\section{Author Contribution}

Katalin Szakszon and Anikó Ujfalusi prepared the manuscript. Anikó Ujfalusi supervised the molecular cytogenetic studies of the patients. Katalin Szakszon is also the clinical geneticist who performed clinical assessment, examination, and genetic counseling of the family. Orsolya Nagy and Beáta Bessenyei performed molecular karyotyping and cytogenetic studies. Ádám Borbély was the psychiatrist of patient 2, and Györgyi Lente was the psychologist of patient 1; they assessed the behavioral and intellectual characteristics. Irén Kántor was the endocrinologist who also raised the suspicion of a possible genetic cause of the symptoms. All authors took part in revising the relevant sections.

\section{References}

Ahn K, Gotay N, Andersen TM, Anvari AA, Gochman $\mathrm{P}$, et al: High rate of disease-related copy number variations in childhood onset schizophrenia. Mol Psychiatry 19:568-572 (2014).

- Bendel RP, Baldinger S, Millard C, Arthur DC: Two successive partial trisomies for opposite halves of chromosome 22 in a mother with a balanced translocation. J Med Genet 19:313 (1982).

- Biesecker LG, Rosenberg M, Dziadzio L, Ledbetter DH, Ning Y, et al: Detection of a subtle rearrangement of chromosome 22 using molecular techniques. Am J Med Genet 58:389394 (1995).

- Boyd LJ, Livingston JS, Brown MG, Lawce HJ, Gilhooly JT, et al: Meiotic exchange event within the stalk region of an inverted chromosome 22 results in a recombinant chromosome with duplication of the distal long arm. Am J Med Genet 138:355-360 (2005).

- Chen CH, Chen HI, Liao HM, Chen YJ, Fang JS, et al: Clinical and molecular characterization of three genomic rearrangements at chromosome 22q13.3 associated with autism spectrum disorder. Psychiatr Genet 27:23-33 (2017).

-Choi SY, Pang K, Kim JY, Ryu JR, Kang H, et al: Post-transcriptional regulation of SHANK3 expression by microRNAs related to multiple neuropsychiatric disorders. Mol Brain 8:74 (2015).

-Coutton C, Dieterich K, Satre V, Vieville G, Amblard F, et al: Array-CGH in children with mild intellectual disability: a populationbased study. Eur J Pediatr 174:75-83 (2015).

Failla P, Romano C, Alberti A, Vasta A, Buono S, et al: Schizophrenia in a patient with subtelomeric duplication of chromosome 22q. Clin Genet 71:599-601 (2007).

Feenstra I, Koolen DA, Van der Pas J, Hamel BCJ Mieloo H, et al: Cryptic duplication of the distal segment of $22 \mathrm{q}$ due to a translocation (21; $22)$ : three case reports and a review of the literature. Eur J Med Genet 49:384-395 (2006).
Fryns JP, De Backer D, Lemli L, Pedersen JC, Van den Berghe H: Partial duplication of the long arm of chromosome 22 (22q 13) with complete 22 trisomy phenotype. Acta Paediatr Belg 33:125-127 (1980).

Gardner RJ, Sutherland GR, Shaffer LG: Chromosome Abnormalities and Genetic Counseling (Oxford University Press, Oxford 2011).

-Han K, Holder JL, Schaaf CP, Lu H, Chen H, et al: SHANK3 overexpression causes manic-like behaviour with unique pharmacogenetic properties. Nature 503:72-77 (2013).

Hou JW: Trisomy chromosome (22)(q13.1-qter) as a result of paternal inversion (22)(p11q13.1) proved using region-specific FISH probes. Chang Gung Med J 28:657-661 (2005).

Jafri F, Fink J, Higgins RR, Tervo R: 22q13.32 deletion and duplication and inversion in the same family: a rare occurrence. ISRN Pediatr 2011:829825 (2011).

- Jin C, Zhang Y, Kim S, Kim Y, Lee Y, Han K: Spontaneous seizure and partial lethality of juvenile Shank3-overexpressing mice in C57BL/6 J background. Mol Brain 11:57 (2018).

Johannessen M, Haugen IB, Bakken TL, Braaten $\varnothing$ : A 22q13.33 duplication harbouring the SHANK3 gene: does it cause neuropsychiatric disorders? BMJ Case Rep 12:e228258 (2019).

Koolen DA, Reardon W, Rosser EM, Lacombe D, Hurst JA, et al: Molecular characterisation of patients with subtelomeric $22 \mathrm{q}$ abnormalities using chromosome specific array-based comparative genomic hybridisation. Eur J Hum Genet 13:1019-1024 (2005).

Liang JS, Shimojima K, Yamamoto T: Application of array-based comparative genome hybridization in children with developmental delay or mental retardation. Pediatr Neonatol 49: 213-217 (2008).

Magri C, Marchin E, Bertini V, Traversa M, Savio $\mathrm{G}$, et al: SNP array and FISH analysis of a proband with a 22q13.2-22qter duplication shed light on the molecular origin of the rearrangement. BMC Med Genet 16:47 (2015).
Okamoto N, Kubota T, Nakamura Y, Murakami R, Nishikubo T, et al: 22q13 microduplication in two patients with common clinical manifestations: a recognizable syndrome? Am J Medi Genet A 143A:2804-2809 (2007).

Peeters H, Vermeesch J, Fryns JP: A cryptic duplication 22q13.31 to qter leads to a distinct phenotype with mental retardation, microcephaly and mild facial dysmorphism. Genet Couns 19:365-371 (2008)

- Petek E, Köstl G, Mutz I, Wagner K, Kroisel PM: Characterization of a de novo partial trisomy 22q13-qter in a patient by microFISH. Clin Dysmorphol 9:55-57 (2000).

- Pramparo T, De Gregori M, Gimelli S, Ciccone R, Frondizi D, et al: A $7 \mathrm{Mb}$ duplication at 22q13 in a girl with bipolar disorder and hippocampal malformation. Am J Med Genet A 146A:1754-1760 (2008).

Rahikkala E, Forsström LM, Kokkonen H, Knuutila S, Mustonen A, Ignatius J: Report of interstitial 22q13.1q13.2 microduplication in two siblings with distinctive dysmorphic features, heart defect and mental retardation. Eur J Med Genet 56:389-396 (2013).

-Samanich J, Montagna C, Morrow BE, Babcock $\mathrm{M}$ : Interstitial duplication of $22 \mathrm{q} 13.2$ in a girl with short stature, impaired speech and language, and dysmorphism. J Pediatr Genet 1: 47-53 (2012).

Schinzel A: Incomplete trisomy 22.II. Familial trisomy of the distal segment of chromosome $22 \mathrm{q}$ in two brothers from a mother with a translocation, $\mathrm{t}(6 ; 22)(\mathrm{q} 27 ; \mathrm{q} 13)$. Hum Genet 56:263-268 (1981)

Shimojima K, Tanaka K, Yamamoto T: A de novo intra-chromosomal tandem duplication at $22 q 13.1 \mathrm{q} 13.31$ including the Rubinstein-Taybi region but with no bipolar disorder. Am J Med Genet A 149A:1359-1363 (2009).

-Wieczorek D, Holtvogt J, Thonig S, GillessenKaesbach G: A female patient with partial duplication 22 (q13 $\rightarrow$ qter). Clin Dysmorphol 7: 289-294 (1998).

Wu Y, Ji T, Wang J, Xiao J, Wang H, et al: Submicroscopic subtelomeric aberrations in Chinese patients with unexplained developmental delay/mental retardation. BMC Med Genet 11:72 (2010). 